\title{
Finite Element Simulation of Dent Resistance for Automotive Rear Bumper
}

\author{
Illker Bahar ${ }^{1 *} \mathbb{D}$ \\ 'TOFAŞ Türk Otomobil Fabrikası A.Ş R\&D Center, Bursa, Turkey
}

\begin{abstract}
In automotive industry, there is an increasing demand for weight reduction. On the other hand extraordinary style for automotive exteriors are used in order to increase number of sales. As a result, the usage of different shapes for exterior bumpers is getting increased. Because of that, finite element simulation of dent resistance for automotive bumpers has become a very important step in early steps of automotive design. In this paper, static dent resistance for automotive exterior rear bumper is investigated by using nonlinear finite element method. The thickness change for different areas of bumper is considered from CAD data. In addition, nonlinear mechanical properties such as plastic behavior of rear bumper material are used in the finite element simulations. In addition, the results that are obtained from physical test are also presented here to compare with the results of finite element simulations to see percentage of correlation. This study is mainly focused on the correlation between finite element simulations and physical test results. The finite element simulation results show us that the percentage of correlation with the physical test results is reasonable. Consequently, due to high percentage of correlation between nonlinear finite element results and physical test results, the nonlinear finite element results should be taken into account in early steps of automotive design in order to reach minimum cost and get the most robust solution to eliminate quality problems which may occur in the future.
\end{abstract}

Keywords: Automotive exterior bumper, denting resistance, hyperelastic material, nonlinear finite element simulation, static analysis.

\section{INTRODUCTION}

Nowadays, development of automotive industry leans on using more light weighted materials and production processes as well known to decrease emission rates. On the other hand, there is a greatest challenge at this side an automobile should be not only safer, more comfortable but also different style lines in order to get a step forward. According to grip this value commonly, the usage of different type shapes and material in automotive exterior front and rear bumper is increasing $[1,2,3]$.

In open literature, the studies that are related with finite element simulations of dent resistance for automotive panels and correlation studies between simulations and experiments can be found. Dylan et al. compared between experimental results and finite element simulations in terms of static and dynamic denting. They dented panels with various thickness by using a steel ball. They used LS-Dyna and Abaqus for finite element simulations. At the end of study, they shown that is important using correct material data, the best suitable element formulations, hardening effects and mesh quality [4]. Vreede et al. investigated influence of material properties and geometric shape of panel on their study. Also, they compared between finite element simulation results and experimental results [5]. Sakai et al. analyzed the stiffness of an automotive outer surface panel from both aspects of finite element analyses and experimental results in their study. Their study shows that the stiffness is influenced from rigidity areas and the characteristic of denting is stronger in surfaces with small radius [6]. Holmerg and Nejabat studied on automotive side door's stiffness and dent properties. They analyzed side door in two steps by using finite elements simulations. At first step, the stamping effects were not taken into account but, at second step vice versa. They showed the accuracy level of simulations with stamping effect in their study [7]. Heckmann et al. study on approach that reduces sheet thickness and subsequent of weight automotive thin panel sheets by increasing denting resistance of them. They investigated extrinsic stress on the components should be produced in a dedicatedly aligned shape [8]. Jeong developed a design software that uses empirical equations to predict denting stiffness value of panels. 
By using this, an optimization period is used in early phases development of an automotive [9].

This study shows an objective measurement and consistent correlation level of static dent resistance for exterior rear bumper that used an automotive with new design. The higher percentage of correlation leads virtual analysis team and design team to achieve the robust solution in early steps of design processes. Respectively, an automobile rear bumper has been investigated in terms of targets that defined in physical test and virtual analysis norms. Moreover, mechanical properties of bumper's material were obtained from test results. Because of production and material, same areas have different thickness ratio compared to other. So, all thicknesses were included to create correct finite element model. At the end of the study, physical test results and finite element simulation results were evaluated and compared.

\section{MATERIAL AND METHOD}

As known from the literature, the FEMs generated in solvers are usually nonlinear and can involve from a few to thousands of variables. In terms of these variables the equilibrium equations obtained by discretizing the virtual work equation can be written symbolically as Eq. (1) below [14]:

$$
\mathrm{F}^{\mathrm{N}}\left(\mathrm{u}^{\mathrm{M}}\right)=0
$$

Where is the force component conjugate to the variable in the problem and is the value of the variable. Many of the simulations are history dependent, so the solution must be developed by a series of "small" increments. Two issues arise: how the discrete few to thousands of variables equilibrium statement Eq. (1) is to be solved at each increment, and how the increment size is chosen. Generally, finite element solvers use Newton's method as a numerical technique for solving the nonlinear equilibrium equations. The motivation for this choice is primarily the convergence rate obtained by using Newton's method compared to the convergence rates exhibited by alternate methods such as modified Newton or quasi-Newton methods for the types of nonlinear problems. The basic formalism of Newton's method is as Eq. (2) follows [13]:

$$
x_{1}=x_{0}-\frac{f\left(x_{0}\right)}{f^{\prime}\left(x_{0}\right)}
$$

This process may be repeated as many times as necessary to get the desired accuracy. In general, for any $x$-value , the next value given by Eq. (3) below [14]:

$$
x_{n+1}=x_{n}-\frac{f\left(x_{n}\right)}{f^{\prime}\left(x_{n}\right)}
$$

What Newton's method actually does can be seen from Fig. 1-a:

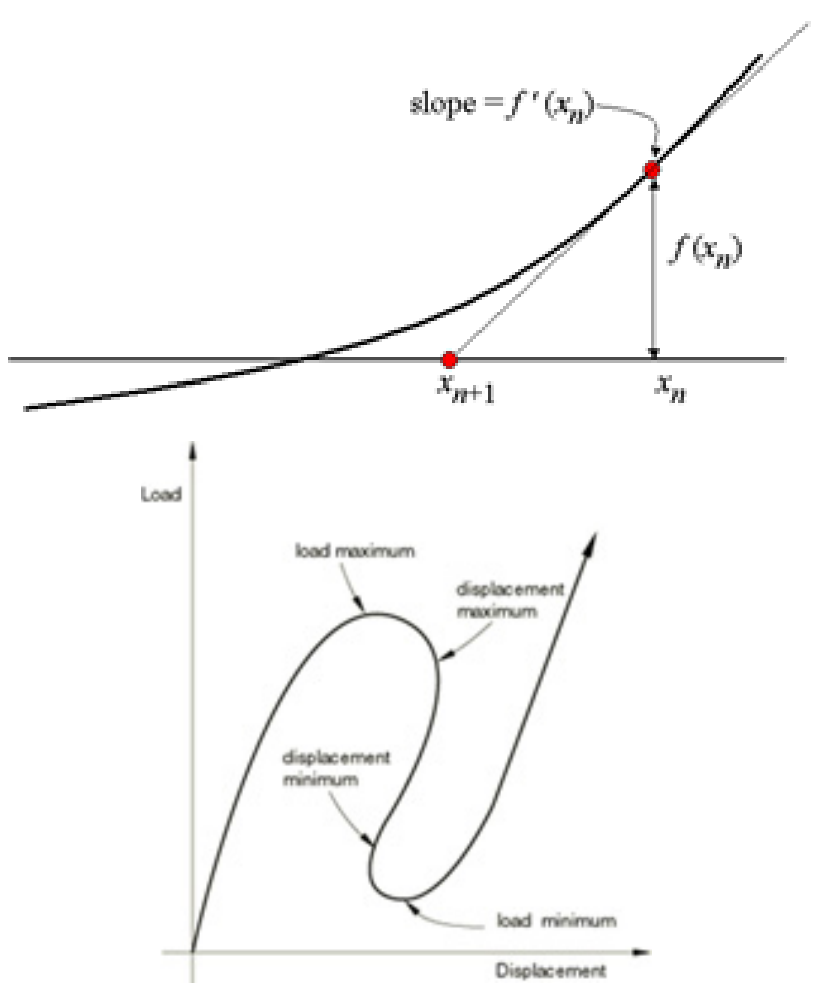

Figure 1. a) Demonstrate of Newton's Method, b) Typical unstable static response

Dent resistance simulations are usually unstable problems due to buckling. It is often necessary to obtain nonlinear static equilibrium solutions for unstable problems, where the load-displacement response can exhibit the type of behavior sketched in Fig. 1-b. During periods of the response, the load and/or the displacement may decrease as the solution evolves. The modified Riks method is an algorithm that allows effective solution of such cases It is assumed that the response is reasonably smooth that sudden bifurcations do not occur. The basic algorithm remains the Newton method; therefore, at any time there will be a finite radius of convergence. Further, many of the materials (and possibly loadings) of interest will have path dependent response. For these reasons, it is essential to limit the increment size. In the modified Riks algorithm, along the tangent line to the current solution point and then searching for equilibrium in the plane that passes through the point thus obtained and that is orthogonal to the same tangent line [5]. In this study, the Newton method which is implemented in Abaqus was used.

\section{NUMERICAL STUDIES}

Loading conditions, constraints, thickness and material properties are critical parameters to be able to solve complicated denting resistance and complicated behavior of buckling. All of them should be defined correct. FCA technical terms that used during the whole study can be found at Table 1.

Detailed FEM of rear bumper is created to perform nonlinear buckling analyses mesh detail of rear bumper can be seen easily from Figure 2 . 


\begin{tabular}{|c|c|}
\hline \multicolumn{2}{|c|}{ Table 1. Technical terms } \\
\hline Technical Terms & Explanation \\
\hline Buckling & Recoverable, localized reversal of curvature failure \\
\hline Permanent Dent & Residual visible deformation \\
\hline Deflection & Maximum deformation at full load application. \\
\hline Zone B & Zone name for main bumper area. \\
\hline Zone C & Zone name large-unsupported area of bumper. \\
\hline Zone D & Zone name for wheel openings of bumper. \\
\hline Zone E & Zone name for body joints of bumper. \\
\hline Zone F & Zone name for step pad area of bumper. \\
\hline Zone I & Zone name for backup \& read fog light lens of bumper. \\
\hline
\end{tabular}

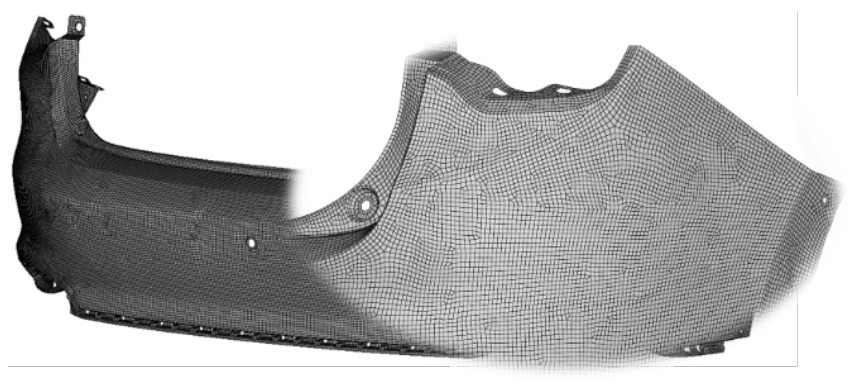

Figure 2. Mesh Structure of Rear Bumper

Generally, FEM of an automotive BIW is modeled with shell elements. Because, almost $95 \%$ of an automotive structure created from thin sheet plates. So, they can be modeled by using shell theorem as Figure 3-a. Adhesives and other parts which are not suitable for shell theorem are modelled with 3D solid continuum elements (non-reduce) which can be seen from Figure 3-b. Morover, fasteners such as spot welds are modeled as point element. They had beam section which is rod attribute with $5 \mathrm{~mm}$. Bolt connections are modeled with rigid elements which are constrained in all degree of freedoms from independent nodes.

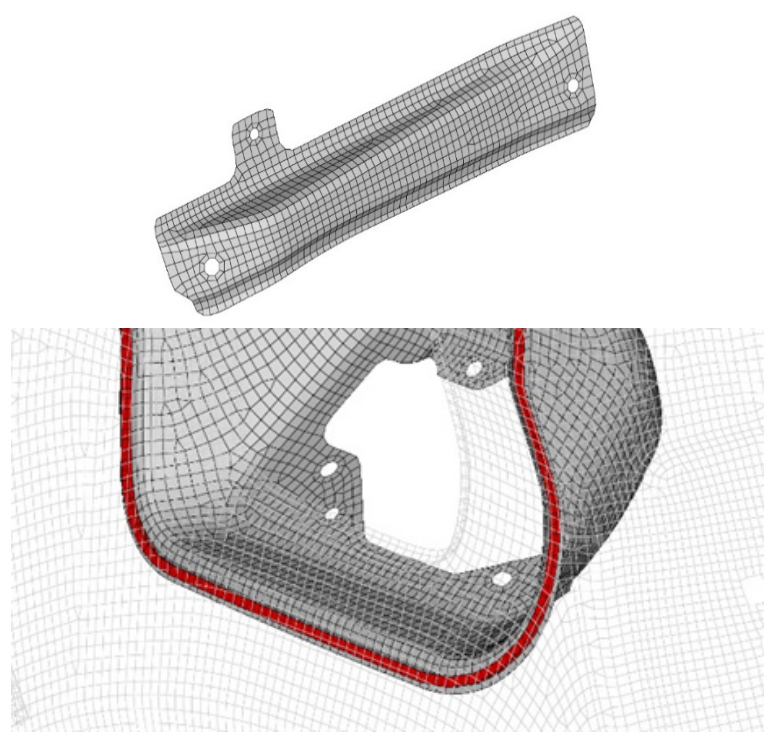

Figure 3. a) 2D Modelling of $F E$, b) 3D Modelling of $F E$

The indenter which consist of a cylindrical pusher and a cover is modelled by using 3D solid continuum elements which are hybrids (hyperelastic material) for applying force to bumper surface in tangent direction. The cylindrical pusher has $80 \mathrm{~mm}$. diameter top hat. The indenter cover is 7.5 $\mathrm{mm}$ thick and made from high-density foam sheet. Detailed
FEM of the indenter can be seen from Figure 4.

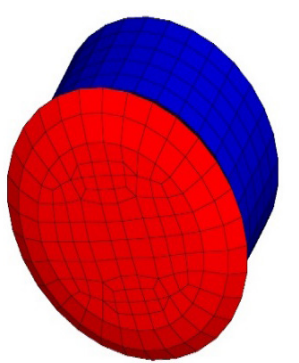

Figure 4. FEM of the Indenter

Element quality is very important for finite element analyses. Especially, it can be main problem for convergence ratio such as buckling analysis which are highly non-linear [15]. It's clear that using quad elements instead of triangle elements always gives better results. So, equality for whole model shown at Table 2 below.

Table 2. Summary of element quality

\begin{tabular}{|c|c|c|}
\hline & Value & Pass Criteria \\
\hline Tria Ratio for Comps & 4.8 & $\leq 5.0$ \\
\hline Maximum Warpage & 19 & $\leq 20.0$ \\
\hline Aspect Ratio & 2.8 & $\leq 3.0$ \\
\hline Skewness & 42.2 & $\leq 45.0$ \\
\hline Jacobian & 0.61 & $\geq 0.6$ \\
\hline
\end{tabular}

Usage of correct material properties for nonlinear analysis is so important in case a target which is defined in terms of number such as deformation [16]. Thus, the rear bumper's material which is PP 48.380 modelled as isotropic behavior with included plastic behavior that extracted from test results. PP 48.380 is a Polypropylene which is filled with mineral (talc). The material of parts for half model are also included with material which have detail behavior for this analysis. All sheet's material properties are extracted form tensile tests and applied for each steel part separately. The used materials are FEP02, FEP04, FEP05, FE180BH, FE210IF, FE 600DP, FE 1000DP which are introduced according to FCA Norms [15].

The indenter's material has a hyperelastic behavior in simulations. So, it is important to usage of correct material for indenter. When the indenter pushed to rigid wall, the behavior of the intender pusher and the indenter of cover act like graph below. Shape change of the indenter under force also can be seen from Graph 1. Wireframe with blue color represents initial position of the indenter and red color represents deformed shape of the indenter.

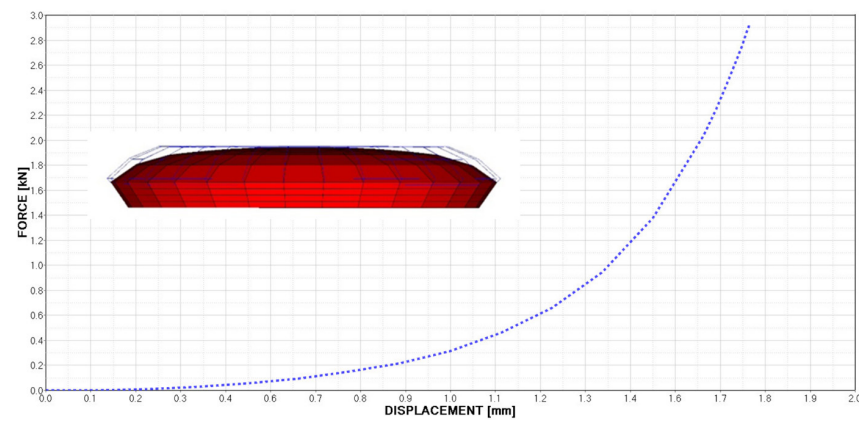

Graph 1. Behavior of the indenter 
Dent resistance problems are local issue problems. So that in order to decrease computation time, half FEM is used as Figure 5-a. Intersection nodes which lean on cutline are constrained in all degree of freedoms. Loading conditions for indenter are shown at Figure 5-b. Indenter is positioned to tangent of bumper surface and load applied in local normal to indenter. Indenter constrained all degree of freedoms except normal direction of surface tangent. When the force reached to target value, the force is removed in axial direction and indenter moved from current position back to initial position.
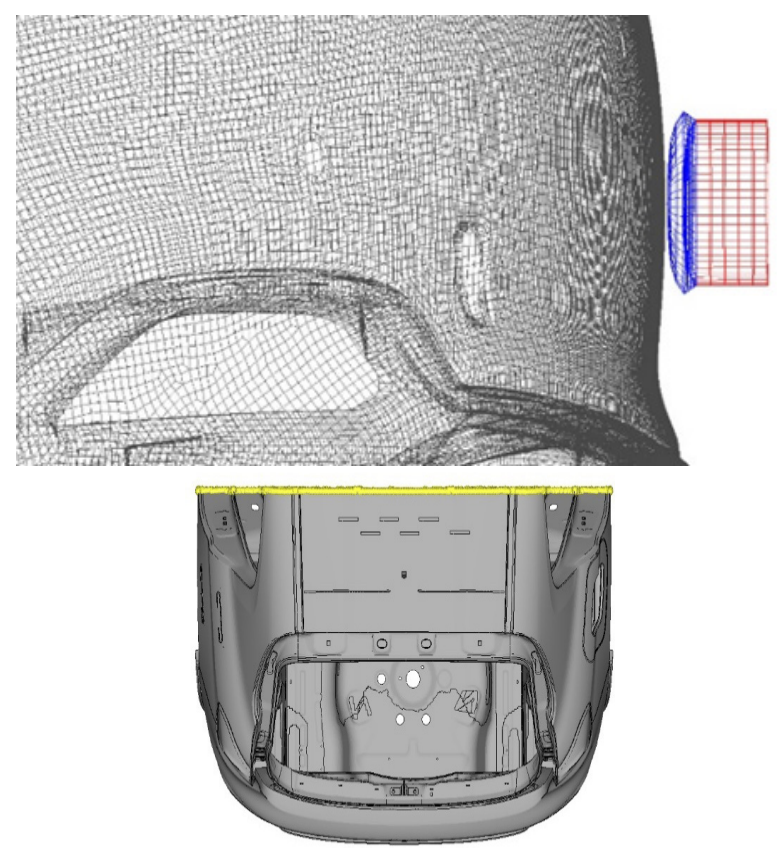

Figure 5. a) Boundry conditions b) Loading conditions

Bumper's area divided to specific zones which are described in norms [16]. These zones have no specific loading points, but all zones have different pre-defined pass criteria which are described in Table 1. According to loading zones, the indenter is positioned. Loading points of the indenter shown at Figure 6. Only loading point with red circle which leans on Zone B (Main Bumper) is selected for this study.

FEM is prepared on Hypermesh 2017.2.3 and solved by using ABAQUS 6.14 in nonlinear implicit solver. Results post-processed by using Hyperview 2017.2.3. Similar to $[10,11,12], \mathrm{U}, \mathrm{S}$, PEEQ and RF are requested for the results and plotted for graphs. Maximum displacement for loading can be seen from Figure 7-a. Pass criteria is defined as maximum displacement should be lower than $15 \mathrm{~mm}$. Permanent displacement with small denting after removing load slowly is shown at Figure 7-b. Permanent displacement according to pass criteria should be lower than $1 \mathrm{~mm}$. It is also known that dents higher than $0.5 \mathrm{~mm}$ can be seen easily from anyone.

According to finite element result, force-displacement is plotted by using reference node values of the indenter and the reference node values of bumper. From Graph 2, the stiffness change can be seen at $4.5 \mathrm{~mm}$ and $12 \mathrm{~mm}$ displace- ments. In addition, graph has similar zones that described in Fig. 1-b except the buckling behavior.

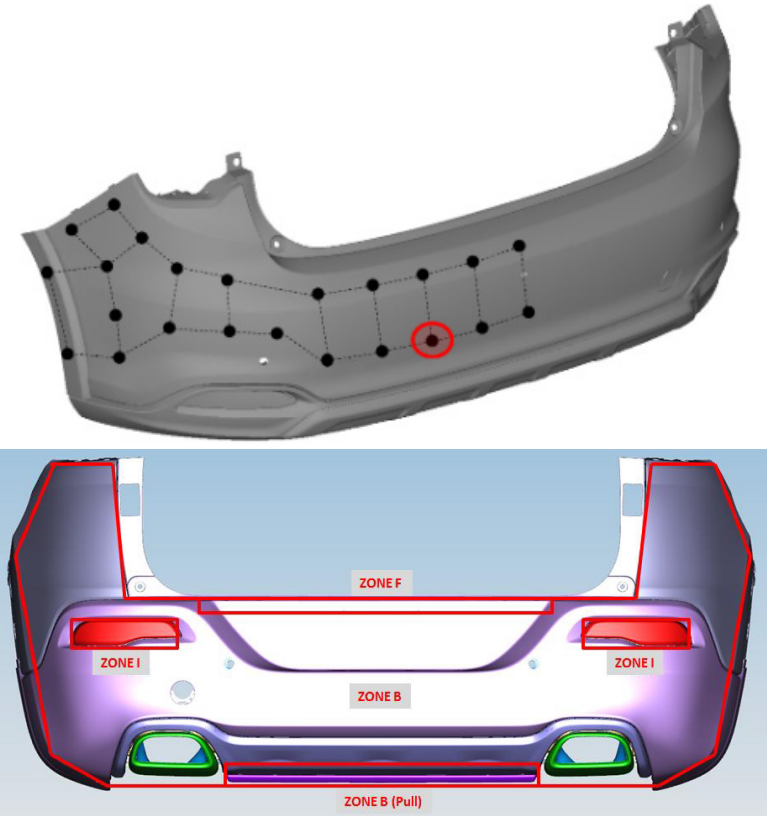

Figure 6. Indenter positions for bumper according to zones

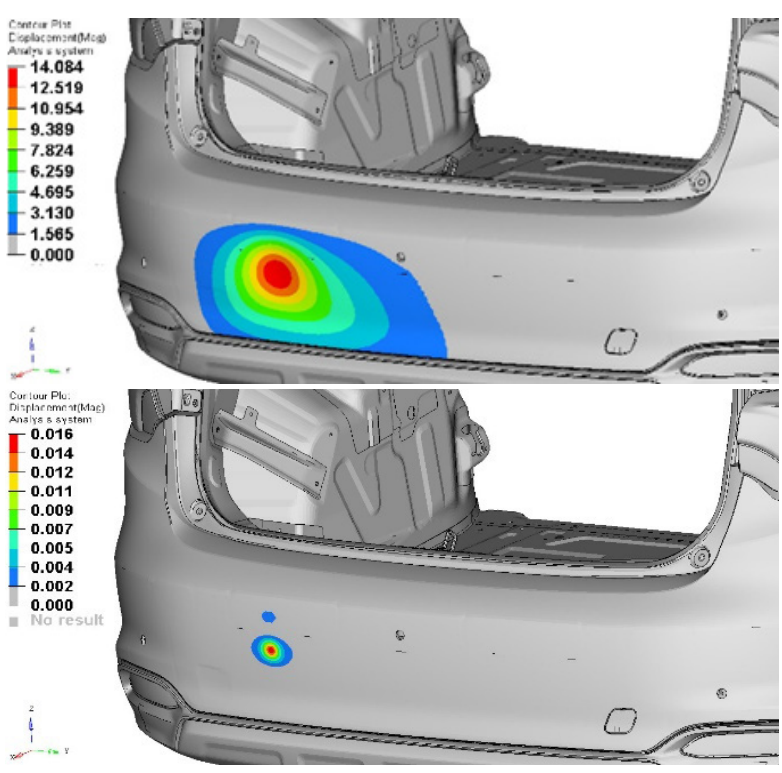

Figure 7. a) Maximum displacement contour b) Permanent displacement contour

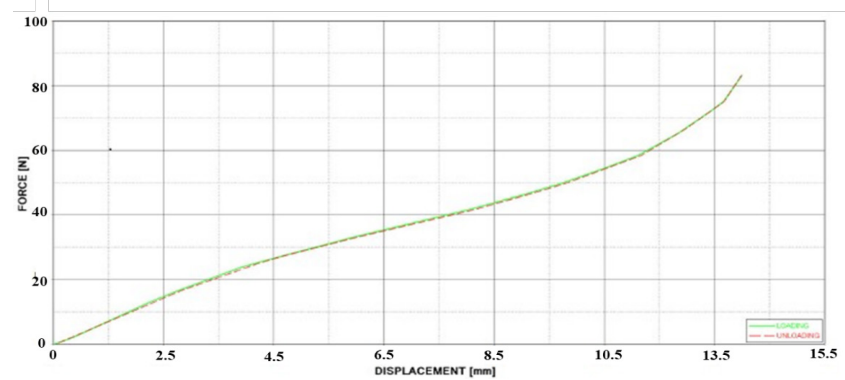

Graph 2. Force - displacement curve for current point extracted from analysis

\section{EXPERIMENTAL VERIFICATIONS}

The test vehicle is connected to a fixture which is rigid. A 
general application can be seen from Figure 8-a. The load is applied from indenter according FCA test norm and removed in same axial direction which is normal to surface back. The maximum and permanent deformations are measured from linear displacement transducer. Also, reaction force of indenter is read from load cell as shown Figure 8-a. Loading point assessments for physical test setup shown at Figure 9-b. They are positioned to almost same location as FEM.

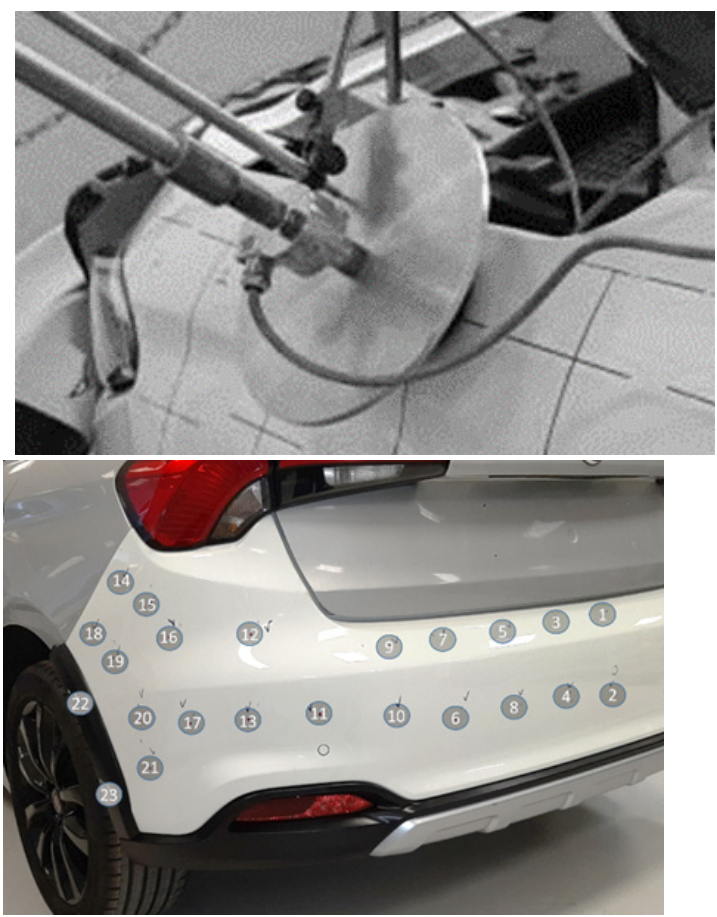

Figure 8. a) Typical physical test setup for denting resistance b) Loading point assessments

Physical test result for current position of indenter can be seen clearly from at Graph 3 below. Stiffness changes which is result of contact between parts can be extracted from loading - unloading curves. In addition, no buckling behavior is seen in physical test result as finite element simulation. On the other hand, some differences can be extracted from graphs between physical test result and finite element simulation result.

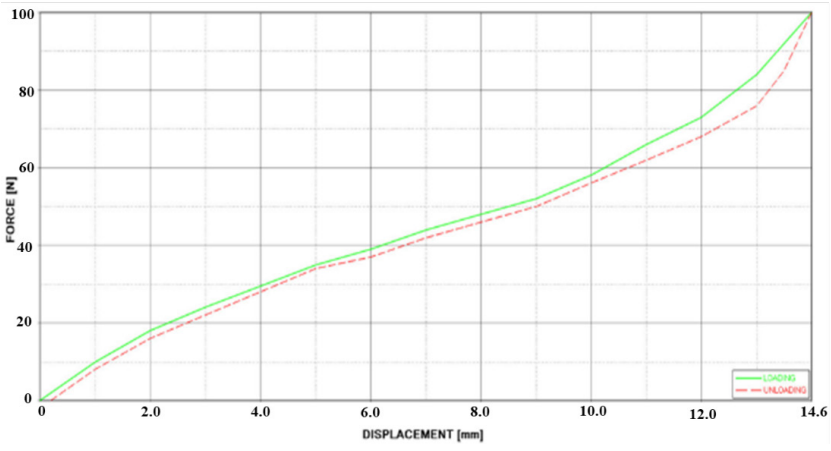

Graph 3. Force - displacement curve for current point extracted from physical test

\section{DISCUSSIONS}

The percentage of correlation status between finite element analysis and physical test is investigated by using force-displacement of actual point for loading and unloading con- ditions. Maximum and permanent deformations under static test load are so important to specify performance of a bumper. These parameters are also related with stiffness of bumper.

The other important thing is the buckling which is related with quality in terms of visible denting. As a result, several physical tests should be repeated in order to reach targets. Furthermore, it is required more prototype which means not only higher cost but also more waste of time. The better correlation between finite element simulations means that the less cost and waste of time.

Getting high percentage correlation is not easy at first step. In other words, some iterations are needed. For example, Figure 9 shows us location difference between test (blue) and finite element simulation (red). At first step, the distance between two location is $20 \mathrm{~mm}$ in direction-y and 10 $\mathrm{mm}$ in direction-z. Although they are small distances, they cannot be ignored. In this scenario, the maximum displacement difference is $1.86 \mathrm{~mm}$ and the permanent set difference is $0.29 \mathrm{~mm}$.

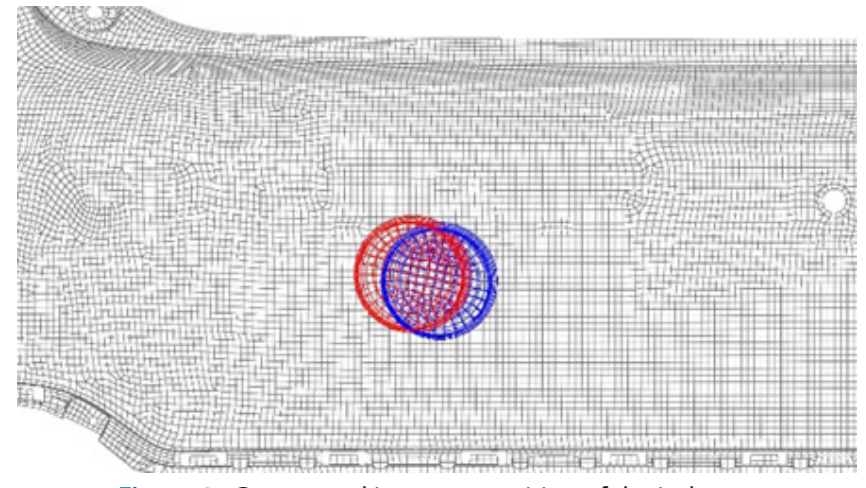

Figure 9. Correct and incorrect position of the indenter

The other issue is material properties and thickness. In this study material has been modeled like isotropic but, PP materials should be modeled composite in order to correct behavior. No action is taken for this issue because of necessary programs that are used for modeling composite materials.

Figure 10 shows the distribution of thickness. In early, same thickness has been used for whole model of bumper such as ribs, faces, radius but some areas have not same thickness ratio as other areas due to production processes in terms of molding, angle. Variable thickness ratio for different areas of same component location are so effective for deformations. For example, the maximum displacement difference between unique thickness and variables thickness is $1.22 \mathrm{~mm}$ and the permanent set difference between unique thickness and variables thickness is $0.11 \mathrm{~mm}$.

After investigated issues above, some of them solved such positioning the indenter, correct thickness distribution but, any action taken for material modelling. The percentage of correlation status, between finite element simulation and physical test has been shown by using graph values at Table 3. With solved issues, correlation status got better as much 


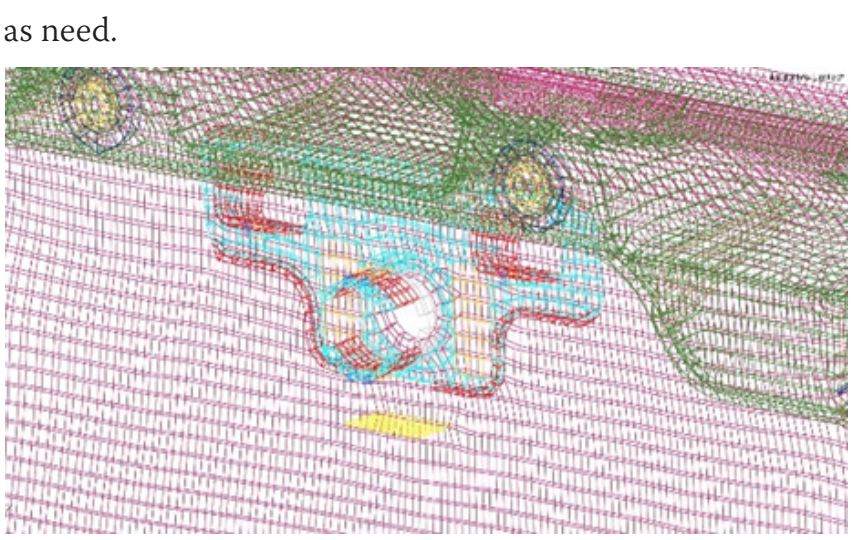

Figure 10. Thickness distribution of bumper's FEM

Table 3. Correlation Status

\begin{tabular}{|c|c|c|c|}
\hline & Test & FEM & Diff \% \\
\hline Maximum Deformation $[\mathrm{mm}]$ & 14.675 & 14.084 & 4.2 \\
\hline Permanent Deformation $[\mathrm{mm}]$ & 0,020 & 0.016 & 25 \\
\hline Visible Denting & NO & NO & 0.0 \\
\hline
\end{tabular}

\section{CONCLUSION}

In this study, static dent resistance for automotive exterior rear bumper investigated by using nonlinear finite element method. In addition, the results that are obtained from physical test are also presented here to compare with the results of finite element simulations to see percentage of correlation. Results can be summarized below;

- All loading points should have positioned to exactly same location in both psychical tests and finite element simulations. A small different between positions effects results so that the percentage of correlation get worse.

- Not only bumper parts but also the other parts that belong to body in white should be into accounted in order to more accurate results. Because, nonlinear contact interactions are important for these type analyses.

- Material properties are also crucial effect for correlation. They should be defined correctly. In this study, PP material defined like Isotropic but composite behavior will effect the results.

- Thickness distribution for plastic parts so important. Because of production, generally plastic parts have different thicknesses on same components. They should be defined properly.

Due to the high coordination, these results show us that the number of prototypes can be reduced and optimization can be made at the same time by using finite element analysis in the early phases of automotive design processes.

\section{NOMENCLATURE}

CAD : Computer aided design

BIW : Body in white

FEM : Finite element model
U : Displacement

S : Stress

PEEQ : Equivalent plastic strain

RF : Reaction force

\section{REFERENCES}

[1] Shi, M., Brindza, J., Michel, P., Bucklin, P. et al. (1997). Static and Dynamic Dent Resistance Performance of Automotive Steel Body Panels. SAE Technical Paper 970158, DOI: https://doi.org/10.4271/970158

[2] Jung, D.W. (2004). A Parametric Study of Sheet Metal Denting Using a Simplified Design Approach. KSME International Journal, 16(12): 1479-1492.

[3] Mahmood, H. F. (1981). Dent Resistance of Surface Panel and Slam Area. SAE Technical Paper No. 810099, ISSN: 0148-7191, e-ISSN: 2688-3627, DOI: https://doi.org/10.4271/810099.

[4] Dylan, T., Hodgins, B., Worswick, M. (2001). Static and Dynamic Denting of Paint Laked aa6111 Panels: Comparison of Finite Element Prediction and Experiments. SAE Technical Paper No. 2001013047, DOI: https://doi.org/10.4271/2001-01-3047.

[5] Vreede, P. T., Tamis, P. J. Roelofsen, M. E. (1995). The Influence of Material Properties and Geometry on Dynamic Dent Resistance: Experiments and Simulations. Proceedings IBEC '95, International Body Engineering Conference, pp. 79-86.

[6] Sakai, H., Saito, K., Tsukada, H. (1983). Stiffness and Dent Characteristics of Body Outer Surface Panel-Finite Element Analysis and Experiment. Int. J. of Vehicle Design, 4(1): 13-22, DOI: 10.1504/ IJVD.1983.061295.

[7] Holmberg, S., Nejabat, B. (2004). Numerical Assessment of Stiffness and Dent Properties of Automotive Exterior Panals. Materials and Design 25: 361-368, DOI: 10.1016/j.matdes.2003.12.005

[8] Heckmann, M., Birkert, A., Scholle, M., Sobhani, M. (2018). Method to Increase Denting Stiffness of Car Body Skin Panels. Journal of Physics Conference Series 1063(1):012089, DOl: 10.1088/17426596/1063/1/012089.

[9] Jeong, D. W. (1983). A Parametric Study of Denting Using a Simplified Design Approach. the Korean Society of Mechanical Engineers, 26(8): 1472-1479, DOI: https://doi.org/10.3795/ KSME-A.2002.26.8.1472.

[10] Zeng, D., Xia, Z. C. (2005). A Comparative Study of Dent Resistance Incorporating Forming Effects. SAE Technical Paper No. 2005010089, DOl: https://doi.org/10.4271/2005-01-0089.

[11] Werner, M. F. (1993). Finite Element Simulation of Steel Body Panel Performance for Quasi-Static Dent Resistance. Automotive Materials - IBEC'93.

[12] Sabbagh, M.A., Chavli, R. N., Montgomery Jay S. (1995). Quasi-Static Dent Depth Simulation Using Nonlinear FEA. IBEC'95 Automotive Body Materials, pp-87-90.

[13] ABAQUS Analysis Theory Manual. ABAQUS Inc., 2017.

[14] ABAQUS Analysis User's Manual. ABAQUS Inc., 2017.

[15] Altair Hyperworks 2017: Users Guide. Altair Engineering, Inc., Troy, Michigan.

[16] FCA Norms 